\title{
L-DOPA-Induced Disinhibition in Parkinson Disease: More than Agonistic
} Behavior

\author{
Thomas Fritsch ${ }^{1,2,3 *}$, Trevor Hyde ${ }^{1}$, Maggie S Wallendal ${ }^{1,3}$ and Paul A Nausieda ${ }^{1}$
}

${ }^{1}$ Parkinson Research Institute of the Wisconsin Parkinson Association, Milwaukee, WI, USA

${ }^{2}$ Department of Neurology Case Western Reserve University School of Medicine, Cleveland, OH, USA

${ }^{3}$ Department of Social Work, Helen Bader School of Social Welfare, University of Wisconsin, Milwaukee, WI, USA

\section{Introduction}

Pharmacologic interventions (levodopa therapy [LVT]; dopamine agonists; $\mathrm{MAO}-\mathrm{B}$ inhibitors; and COMPT-inhibitors) remain the frontline treatment options to manage the motor and non-motor symptoms of Parkinson disease (PD). However, for some patients, treatment with dopaminergic agents, particularly agonist therapies (ATs), can result in pernicious behavioral syndromes classified in the DSM-IV as Impulse Control Disorders (ICDs) [1,2]. ICDs resemble a hypomanic state characterized by uncontrollable urges to engage in behaviors such as excessive gambling, obsessive shopping, hypersexuality, compulsive eating, and punding [1]. Other ICD symptoms include depression, anxiety, and agitation. In a recent study, the prevalence of two forms of ICDs (hypersexuality and gambling) among newly-diagnosed PD patients who were taking therapeutic doses of dopamine agonists was high, $18.4 \%$ [1].

Although it is widely accepted that ATs are the predominant factor in medication-induced ICDs [3] the occasional finding that LVTeither alone, or in combination with an agonist-can cause ICDs requires further examination. Here, we report case study data, derived from objective neuropsychological testing, as well as direct behavioral observations, which seems to suggest that rapidly-increasing, highdosage LVT may be associated with profound neuropsychological and behavioral disinhibition.

\section{Assessment of executive functions (EFs)}

Executive functions were assessed with a battery of valid and reliable instruments. (1) A computer administered version of the Stroop Color-Word Test [4] was used as a measure of selective attention and cognitive flexibility. Color-names are displayed in different colors against a black background-but the color-names and ink colors do not correspond (e.g., the color-name, "red" might be displayed in blue). The subject's task is to ignore the color-names and say the color in which the word is printed. The examinee is given feedback when an error is made and has to correct it before continuing. Two outcome measures result from this task: time to correctly name 20 colors and the number of errors that were made. (2) Verbal Fluency [5] was assessed using semantic and phonemic fluency tasks. In the semantic fluency tasks, subjects are asked to name fruits and vegetables or animals one could find in a zoo. In the phonemic fluency tasks, subjects are asked to produce words beginning with the letters $\mathrm{S}$ or $\mathrm{P}$. The score for each task is total number of items named within one minute. All tests were normatively scored so that results from different tasks could be assessed for equivalence. One semantic and one phonemic task were given at each assessment, and the two task forms were alternated to reduce practice effects. (3) The Alternating Names Test (ANT) [6] is a new bedside test of set-switching, a component of EF. The test was specifically designed for use in persons with PD. Subjects are asked to produce the names of children, switching back and forth from boys' to girls' names. Outcome measures include the time to complete 5 correct pairs and the number of errors made. In development, the ANT demonstrated good convergent and divergent validity. Alternate forms reliability was high for time, although low for errors. (4) The 6 subtests of the Frontal Assessment Battery (FAB) [7] were used to measure conceptualization, mental flexibility, motor programming (successful execution of actions), inhibitory control, and environmental autonomy (excessive dependence on environmental cues).

\section{Case Report}

A 66-year-old, ambidextrous male with 18 years of education was diagnosed with idiopathic PD 3 years after 2-3 years of gait disturbance and 5-6 years of anosmia. Upon his initial intake evaluation, cognitive functioning was high/normal for his age and gender. Executive functions were within the normal range for Parkinson patients.

One year ago, he experienced increasing falls, and this year he demonstrated hypomanic and aggressive behavior, reporting that, "Sometimes I do stupid things." He was never diagnosed with a major depressive disorder, but clinically significant frustration and sadness over his condition were noted. He was reported to our PD clinic staff psychologist by the hospital staff because they felt threatened by his belligerent and possibly aggressive behavior.

The patient underwent neuropsychological testing several times over the course of an observation period (Figure 1). Most notable is the extreme increase in disinhibition that occurred when his LVT was rapidly increased, superimposed over a steady decline in processing speed that appeared related to progressive subcortical pathology-but did not appear to be medication-dependent. Processing speed was correlated with time $(r=-.96)$, but not with levodopa dosage $(r=.25)$. Executive functioning was correlated both with time $(r=-.54)$ and levodopa dosage $(r=-.57)$.

The psychologist also observed that the patient's thought processes seemed to be racing and he was unable to maintain focus in the face of distractors. He appeared disinhibited and impulsive, his reasoning was superficial, and his self-guided insight was poor. He was very apparently overwhelmed by stimuli in the room, unable to concentrate or maintain attention. At this time, the patient met ICD criteria defined in the DSM-IV [2].

Attending neurologists attributed this disinhibition to the rapid increase in LVT. In response, the patient's LVT was rapidly reduced. This change was accompanied by significant improvement in executive functioning and decreased disinhibition (Figure 1).

There is one examination point that does not appear to fit the high-

*Corresponding author: Thomas Fritsch, Parkinson Research Institute, $945 \mathrm{~N}$ $12^{\text {th }}$ Street, Suite 4602, Milwaukee, WI 53233, USA, E-mail: tfritsch.pri@gmail.com

Received May 26, 2013; Accepted August 19, 2013; Published August 21, 2013

Citation: Fritsch T, Hyde T, Wallendal MS, Nausieda PA (2013) L-DOPA-Induced Disinhibition in Parkinson Disease: More than Agonistic Behavior. Biol Syst 2: 115. doi:10.4172/2329-6577.1000115

Copyright: $\odot 2013$ Fritsch T, et al. This is an open-access article distributed under the terms of the Creative Commons Attribution License, which permits unrestricted use, distribution, and reproduction in any medium, provided the original author and source are credited. 


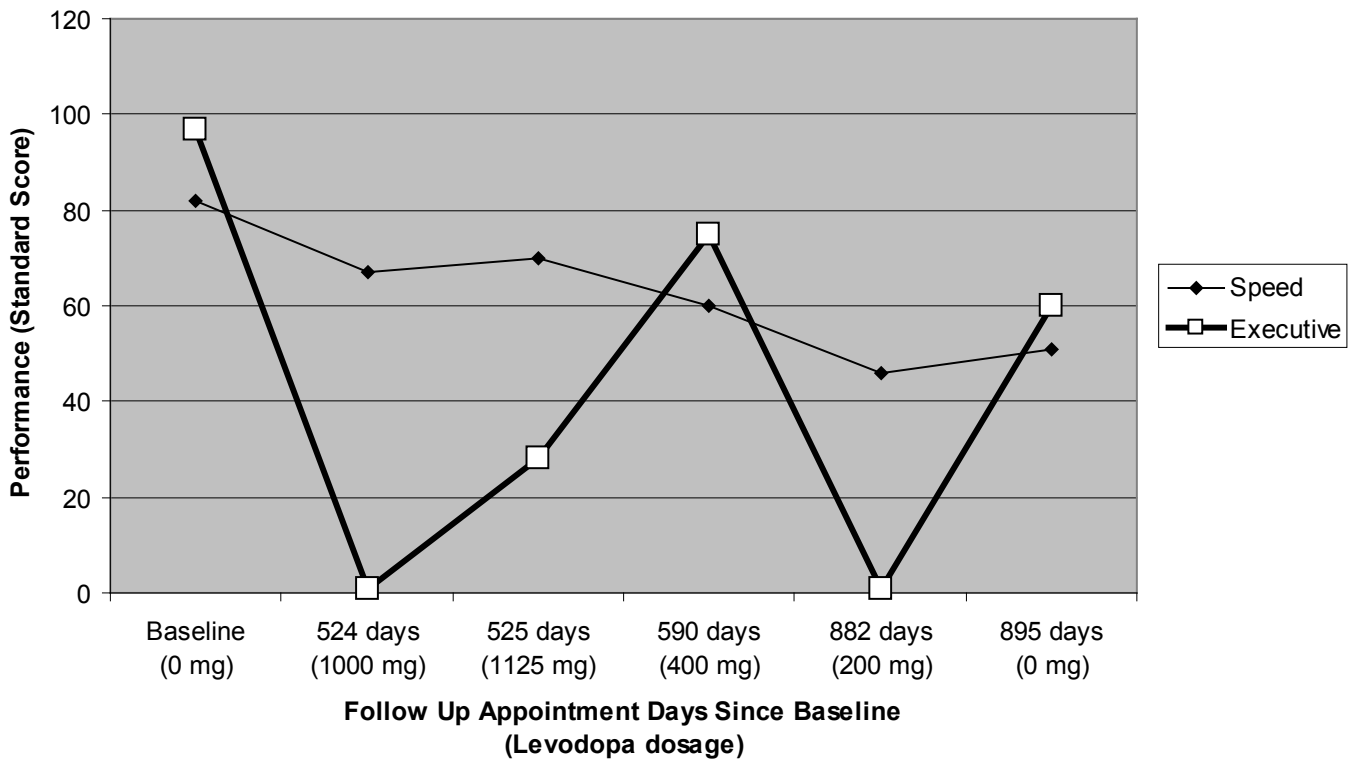

Figure 1: Relationships between disinhibited neuropsychological performance (executive function) and processing speed, with LVT dosages over time.

dose LVT/disinhibition pattern-seen on follow-up day 882 (Figure 1). At this time, the patient was taking relatively low doses of levodopayet still showed severe disinhibition/executive dysfunction. However, during the preceding month his primary care physician had placed him on a medication regimen that was contraindicated by his neurologist. Thus, he was admitted to the hospital in part for medication titration. He was on a daily dosage of $550 \mathrm{mg}$ of levodopa until day 870 , when it was reduced to $400 \mathrm{mg}$. Then, on day 880 , it was reduced to $300 \mathrm{mg}$, and further decreased to $200 \mathrm{mg}$ on day 882 . He was also treated with $100 \mathrm{mg}$ of amantadine and $20 \mathrm{mg}$ of cyclobenzeprine daily until day 880 , when both were discontinued.

\section{Discussion}

To our knowledge, this is among the first case reports showing that rapid, high-dosage increases of LVT can result in significant and detectable neuropsychological disinhibition. Behavioral interactions with our neuropsychologist dovetailed with the objective neuropsychological findings. At his maximum dose, the patient fit the criteria for having an ICD [2]. This finding contradicts reports that only dopamine agonist (DA) therapies are implicated in the development of ICDs [3].

LVT has stimulant effects [8] such as those seen among amphetamine users and alcoholics. The LVT effects on cognition mirror those seen in these amphetamine/alcohol abusers. Although the patient reported on here was also taking a weak indirect DA, this was only prior to the second disinhibited period. Whether the same pattern of results would have been observed if the patient were not on agonist therapy cannot be determined at this time.

In a recent review, Antonelli et al. [9] outline an explanatory framework for ICDs in PD involving three distinct, but interacting components. The authors argued that these components may contribute to instances of cognitive disinhibition: (1) a pre-morbid susceptibility to impulsivity; (2) the contribution of PD disease processes; and (3) the potential effects of therapeutic agents-mainly dopamine agonists. The authors argue that each of these components may contribute to instances of cognitive disinhibition in PD.

\section{Pre-morbid susceptibility to ICDs}

Neuro-imaging studies support the notion of a pre-morbid susceptibility to ICDs [9]. When compared to PD patients without pathological gambling problems, PD patients with pathological gambling problems had abnormal activation in cortical and subcortical areas-brain areas involved in impulse control.

\section{Effects of PD on ICDs}

PD has several variants, and is likely a "family of diseases." This, in turn, complicates efforts to determine how the disease directly (or indirectly) affects impulse control. When using validated tests of gambling problems, there is general consensus that ICDs are not related to socio-demographic characteristics or clinical features of patients with PD without dementia.

Several authors [9] have suggested that the expression of ICDs in PD results from a failure of the negative feedback system that occurs in a subset of patients. In other words, persons fail to anticipate the negative consequences of their actions, rather than overestimating the possible positive outcomes. An interesting hypothesis, but receiving less empirical support, suggests that an "internal clock" is abnormally slow in $\mathrm{PD}$, making immediate rewards more attractive than future rewards. Levodopa therapies or Deep Brain Stimulation seem to correct the problem, per Antonelli and colleagues' [9] review.

\section{Medications and ICDs}

The specific role(s) of medications in ICDs seems to be the least understood area of the three domains in the Antonelli et al. [9] framework. Dopaminergic medications could modulate impulse control at several levels. In one model, it is suggested that use of ATs in patients who are susceptible to ICDs provokes a distorted, positive estimation of gains. Further, any dopaminergic therapy seems to worsen learning from negative outcomes (e.g., immediate losses when gambling).

In a recent study [10] of 3,090 patients with idiopathic PD receiving routine clinical care at 46 movement disorder centers in the United 
Citation: Fritsch T, Hyde T, Wallendal MS, Nausieda PA (2013) L-DOPA-Induced Disinhibition in Parkinson Disease: More than Agonistic Behavior. Biol Syst 2: 115. doi:10.4172/2329-6577.1000115

States and Canada, the overall prevalence of various ICDs was $13.6 \%$ (Compulsive buying in $5.7 \%$, gambling in $5.0 \%$, binge-eating disorder in $4.3 \%$, and compulsive sexual behavior in $3.5 \%$ ). Nearly $4 \%$ had 2 or more ICDs. Given this high prevalence of ICDs in those being treated with agonist therapies, it is incumbent on physicians to monitor patients for signs of ICDs (regardless of cause), keeping in mind that patients often tend to hide the condition or discount it until their lives are seriously affected [11]. Physicians should also be mindful of the possibility that rapidly increased dosing of LVT could also induce cognitive disinhibition.

\section{References}

1. Bostwick JM, Hecksel KA, Stevens SR, Bower JH, Ahlskog JE (2009) Frequency of new-onset pathologic compulsive gambling or hypersexuality after drug treatment of idiopathic Parkinson disease. Mayo Clin Proc 84: 310316 .

2. American Psychiatric Association (2000) Diagnostic and Statistical Manual of Mental Disorders. Fourth Edition. Washington D.C.: American Psychiatric Association.

3. Weintraub D, Siderowf AD, Potenza MN, Goveas J, Morales $\mathrm{KH}$, et al.
(2006) Association of dopamine agonist use with impulse control disorders in Parkinson disease. Arch Neurol 63: 969-973.

4. Strauss E, Sherman EMS, Spreen O (2006) A Compendium of Neuropsychological Tests: Administration, Norms, and Commentary. Oxford: Oxford University Press.

5. Rosen WG (1980) Verbal fluency in aging and dementia. J Clin Neuropsychol 2: $135-146$.

6. Hyde T, Fritsch T (2011) Assessing executive function in Parkinson disease: the alternating names test. Part I. Reliability, validity, and normative data. Parkinsonism Relat Disord 17: 100-105.

7. Dubois B, Slachevsky A, Litvan I, Pillon B (2000) The FAB: a Frontal Assessment Battery at bedside. Neurology 55: 1621-1626.

8. Nausieda PA (1985) Sinemet "abusers". Clin Neuropharmacol 8: 318-327.

9. Antonelli F, Ray N, Strafella AP (2011) Impulsivity and Parkinson's disease: more than just disinhibition. J Neurol Sci 310: 202-207.

10. Weintraub D, Koester J, Potenza MN, Siderowf AD, Stacy M, et al. (2010) Impulse control disorders in Parkinson disease: a cross-sectional study of 3090 patients. Arch Neurol 67: 589-595.

11. Weintraub D (2008) Dopamine and impulse control disorders in Parkinson's disease. Ann Neurol 64: S93-100. 